\title{
The Path and Thinking of Education Reform Driven by Blockchain Technology
}

\author{
Liping $\mathrm{Li}^{\mathrm{a}}$, * \\ School of information, Yunnan University of Finance and Economics, Yunnan, 650221, China. \\ *, a Lipingli@163.com
}

Keywords: Block chain, Education, Innovation.

\begin{abstract}
Block chain is changing the ideas and patterns of global finance, business and public management. In the field of education and teaching, bock chain also shows good application prospects. In order to study the path and method of the application of block chain technology in education reform, this paper analyzes the core technology of the block chain and its advantage in education and teaching, combining with teaching practice, this paper explains the application of block chain in educational innovation in many ways such as block chain transcript, learning certification, individualized learning, learning ledger, teaching resource management, monitoring and evaluation of teaching quality, etc. The article also gives the framework of the block chain teaching platform and puts forward some problems and thoughts of the block chain application used in education and teaching.
\end{abstract}

\section{Introduction}

Blockchain, which is considered as the most important invention of human society since the birth of Internet, has been widely studied in the field of global finance and business services. In the field of education and teaching, blockchains also show good application prospects. However, at present, blockchain technology has not been widely used in the field of education and teaching, and there is still no mature research case, there are still some attacks and potential threats in the blockchain and the blockchain technology talents are also lacking.

Referring to the related literature, in foreign countries, the research on blockchain in education is mainly in blockchain technology teaching and the construction of blockchain teaching platform [1][2]. The domestic research is discussion and communication about big data driven education reform, the application of blockchain in digital society, the computational law, and the development status and application value of Blockchain + Education [3][4][5][6].

The main innovations of this paper are: first, integrate the idea and method of smart contract into the application of learning certification preservation, learning certification, personalized learning and learning ledger. Second, it brings some thoughts to the trust in computing, ethics and data security, and the fundamental problems of education. Third, it tries to expound the concept and practice of blockchain technology with oriental culture, do well the balance between "centralization" and "decentralization", and solve the relationship between "autonomy" and "supervision".

\section{Block Chain's Core Technology and Advantages in Education and Teaching}

Blockchain is a technology that the whole collective maintains a reliable database by decentralization. It uses distributed technology and consensus algorithm to build a trust mechanism. In the blockchain, data is packaged to form a data block, and use cryptography to build associations between data blocks, it is chain. The core idea is distributed, weak center, and autonomous" [3]. In the field of education, the transparency and data cannot be tampered in blockchain system, It is very suitable for credit management, qualification certification, credit granting, employment and promotion, production and study cooperation, etc. [3] 


\subsection{Encryption Technology.}

It uses asymmetric encryption technology, encryption by public key, decryption by private key, the private key is used for signing, and the public key is used for verification. It solves the problem of public transmission of information and key management, allows communication sides to exchange information on unsafe media. blockchain systems generally use the Hash algorithm to quickly verify the user's identity, so that the data can't be changed easily [3]. The above technologies enable the blockchain system to transmit data safely and make data credible. In education and teaching management, it can be used for data confidentiality, educational certification preservation and educational certification.

\subsection{Point to Point Communication Technology.}

$\mathrm{P} 2 \mathrm{P}$ enables all nodes in the network to participate in providing resources, including bandwidth, storage space and computing power, rather than relying on fewer servers [3]. The same information is saved on a number of computers and equipment, which can help solve the single point fault problem and enhance the anti-malfunction ability of the system. In the education and teaching management, P2P technology can not only help to verify the information, but also benefit the average load and stable operation of the whole system.

\subsection{Distributed Consensus Technology.}

By the FLP impossibility theorem, when multiple hosts exchange information in asynchronous communication network, the network is unreliable by default. This requires a mechanism to ensure that each host eventually reaches a consistent state, this is consensus, the purpose is to realize the consistency and uniqueness of the data in each host, and to ensure the normal operation of the system [4]. Distributed technology weakens the center of the system, and each device is self-governing through the smart contract. It solves the problem of the cost and network capacity limit caused by the exponential growth of the number of equipment in the traditional network. For education and teaching management, the consistency and uniqueness of data ensure the accuracy, credibility and effectiveness of teaching materials and learner's data, and smart contracts automate learning certification.

\section{The Path of Educational Innovation Driven by Block Chain}

In education and teaching, we can abstract the activities of education into the interaction of people and educational resources, people such as teachers, students, managers [5]. The person growth and the changes of education resources in the education such as increasing, transformation, flowing and the change of attribute value are built as the blockchains which are the basic transactions, and it should be integrated with large data, cloud computing, Internet of things, artificial intelligence to build the blockchain teaching platform of partial decentralization and mixed deployment model [6]. The teaching platform of blockchain is divided into network layer, management layer, service layer and application layer. Application layer can meet all kinds of application requirements. The service layer allows users to apply blockchain services under system monitoring and provide encapsulated data blocks for different users. Management layer uses smart contract to agree commitments among various elements, as well as agreements between contracting parties performing these commitments, when the conditions are satisfied, the contract will be executed automatically to realize the system autonomy; the network layer uses distributed and consensus technology to build P2P(peer-to-peer) networks [7]. blockchain teaching platform is based on bockchain cloud, and blockchain cloud provides data blockchains and other hardware and software resources, services and applications. The blockchain teaching platform framework is shown in Figure 1. 


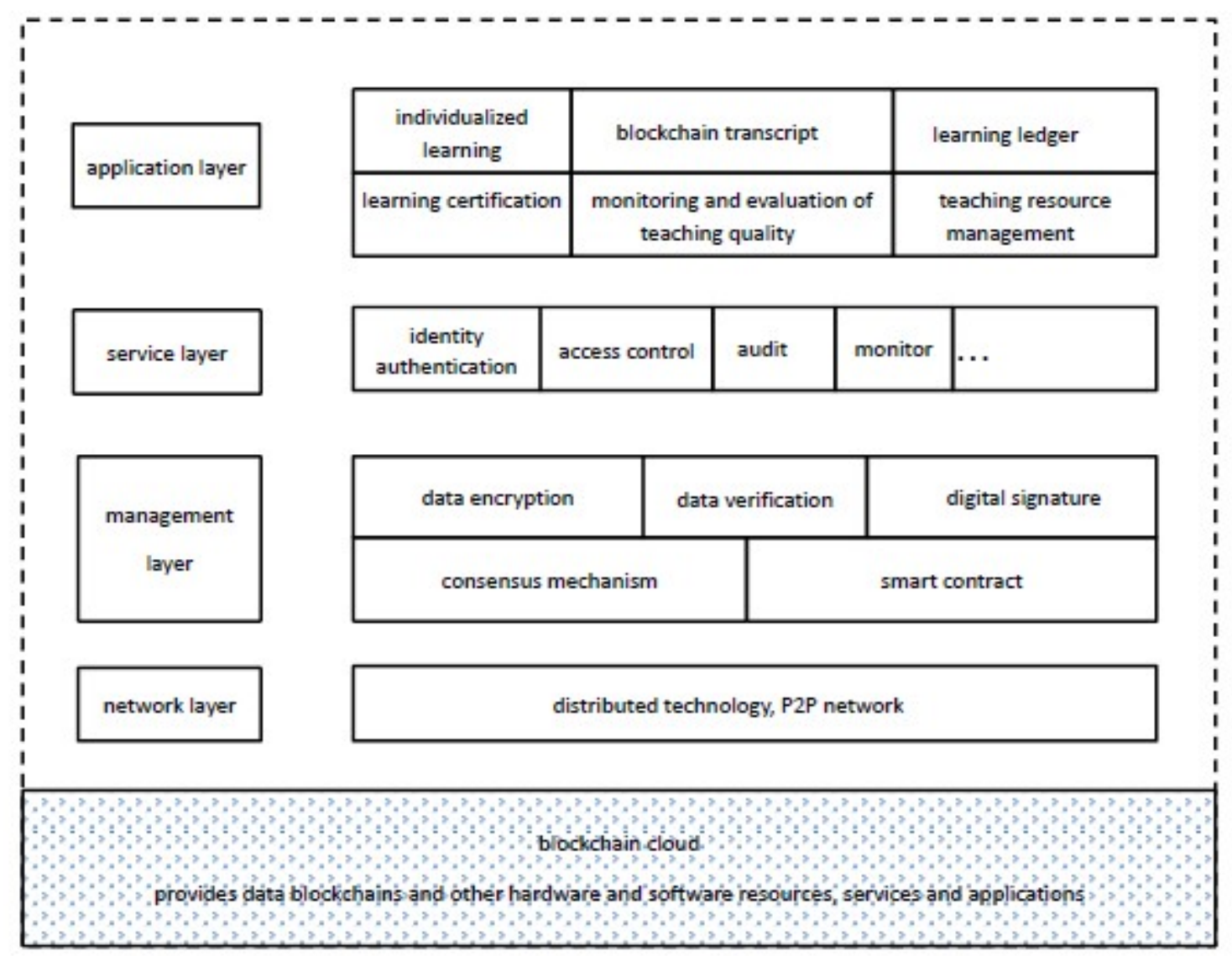

\subsection{Blockchain Transcript}

Figure 1. The blockchain teaching platform framework

The block chain records the learning process and result of learners, it fully presents the detailed data of learners' learning courses, skills, completed learning projects, and credits, evaluations, certifications and so on throughout their lives, this is the blockchain transcript [8]. It can show the learners' learning experience and specialty and provide reliable information for the employers; it is convenient for learners to study and transfer and it does not require relevant schools to provide the proof of learning, the proof of credit, report card and so on. This technology can also help learners create new trust networks, discover other learners' knowledge and skills, help them build the social network system based on learning processes and results [9].

\subsection{Learning Certification}

Economic globalization makes the flow of talents frequent, but at present, the educational certification between different countries and different institutions cannot be well docked, which affects the exertion of talent efficiency. Furthermore, fake diplomas have long been criticized for the system of current learning certification. The use of blockchain technology: First, by blockchain network covering all over the world, learners can study online or offline in different countries and institutions, get real and reliable achievements or certification, and keep their achievements or certification results permanently and safely in blockchains. The third-party employer evaluates the learners' performance or certification to determine whether they are employed. Second, the blockchain network platform can integrate credits, achievements, certificates that obtained by learners from different countries and institutions or make the credit exchange. In this case, the learner may apply for the degrees or certificates to the institutions that recognize this educational model, it can help more people to obtain learning certification and improve the competitiveness of the individual in the labor market. Third, learners and educational institutions can use the smart contract on blockchain education platform to stipulate their responsibilities and obligations, pay and harvest, after completing the learning task in accordance with the provisions of the contract, the learner can 
automatically obtain the corresponding scores or certification. Smart contracts make learning certification more convenient and efficient.

\subsection{Individualized Learning}

First, the block chain tracks and records the learning experience and learning effectiveness of the learners in detail, which helps educators to analyze all kinds of information of learners, predict their habits, preferences, learning modes, etc., and design individualized teaching programs for each learners' educational background and educational needs. Second, with the popularization of mobile electronic devices and the learning environment of technology empowerment is becoming more and more perfect, the time, place and content of learning are becoming more and more decentralization, autonomy. Fragmentation and informal learning are increasingly rising. The blockchain learning platform allows learners to use various mobile electronic devices learning different courses or knowledge at different times and places, and automatically obtain scores or certificates according to the protocol of the smart contracts. It liberates learners from systems, tools, and contents of centralization.

\subsection{Learning Ledger}

Institute for the Future and ACT Foundation jointly put forward the concept of learning ledger, their view is that learning is income. Each learning experience and result of the learner is recorded in the blockchain and becomes a permanent record of the learning ledgers which is increasing constantly and can't be changed easily. The learner can earn credit or certification from an educational institution or other organization. Learning ledgers can track all the knowledge and skills that the learners have learned in the blockchain. In addition, the data in the learning ledgers can be used for analysis to predict which knowledge or skills can bring more benefits to the learners, and learners can also make use of the ledgers to seek their learning investors, the responsibilities, rights, and benefits of both the learners and the investors are agreed and executed by the smart contracts. [10]

\subsection{Teaching Resource Management}

Because of the different needs of each learner, the problem of the customization of teaching resources is put forward. Curriculum resources such as textbooks, teaching videos, teaching plans, question databases, examination papers, teaching materials and teaching cases, etc., and teaching research resources such as the achievements and applications are organized and stored in the way of data blocks and chain structures. Learners can obtain resources on demand and can also produce the blockchain on their own initiative or in response to the needs of other people in order to provide new learning resources for other learners, so as to realize the customization of teaching resources and the model of sharing autonomous learning. The decentralization and co-maintenance of the blockchain make every teacher and student in the system have the opportunity to participate in the management and maintenance of the teaching resources. Collective intelligence optimizes the management of teaching resources. Data encryption and consensus technology can guarantee the credibility of knowledge and protect the intellectual property.

\subsection{Monitoring and Evaluation of Teaching Quality}

With the continuous promotion of education and teaching reform, the monitoring and evaluation of the education and teaching quality is also evolving in understanding and ideas. It needs the organic integration of monitoring and evaluation in the whole process of education and teaching, and blockchains can meet this demand. The subject and professional situation, curriculum settings, contents and requirements of every course, even every knowledge point, as well as the learning process and progress, learning effectiveness and feedback of students can be presented in the blockchain. The information is open and transparent, and they reflect the whole process of teaching in a true and complete way. Because of the weak center and distributed characteristics of the blockchain, there is no single, centralized authority institution of education monitoring and evaluation. The whole society can take part in the supervision of education and teaching in every aspect, put forward useful suggestions, provide effective resources, make deep communication and interaction between schools and society, and improve the quality of education and teaching in an all-round way. 


\section{The Problem and Thinking of the Block Chain Used in Education and Teaching}

\subsection{Computing Trust and Traditional Trust}

The application of block chain in education and teaching is based on computational trust rather than traditional social trust [11], that is, the dependence, agreement and commitment among all subjects will be realized by computer program. Therefore, the mechanism of removing the center and removing the trust is bound to trigger changes in traditional trust mechanisms centered on government agencies and educational authorities. Who has the right of certification? Who will verify it? How to verify? In addition, we need to think about the relationship between smart contracts and the law, the fairness and security of smart contracts, and establishing the business model, and so on. Therefore, governments, education authorities, schools, law departments and all circles of society need to reach consensus and establish the open, fair and impartial trust mechanism based on blockchain.

\subsection{Ethic and Data Security}

With the development of educational big data and educational blockchain concept and technology, the data analysis, processing and utilization are of great significance for scientific management of education and teaching resources, innovation of education and teaching methods, and formulation of education and teaching strategies, but at the same time, the ethical issues and data security problems in the use of data are becoming increasingly prominent. Because the learning experience, marks of students, the teaching cases, examination paper, teaching research of teachers, the data, material, experimental data of courses, and certification information, etc. are all kept in open and transparent blockchains. So, the management of confidential and private data, the protection of intellectual property, and the division of the rights, responsibilities and obligations of all parties in the process of data use are the matter of top priority. We should make high technology serve education in the context of social ethics.

\subsection{The Root of Education}

The application of science and technology is only the means of education, not the main part and the purpose of education and it is also not a tool of knowledge utilitarianism and a gimmick that attract people's eyeballs. blockchain brings us the flexibility and convenience of knowledge acquisition, the individualization of learning, a variety of ways of credit and certificate granting, the trade-off between learning investment and return, etc. But these may also give the opportunities to a few illegal transactions or encourage utilitarian learning. We should establish the perfect guarantee mechanism and make use of the blockchain technology to realistically realize the reform of education and teaching, so that science and technology can serve the cause of educating people better.

\section{Summary}

The block chain, which is regarded as a technology that trigger the fifth social changes in human society will bring great innovation to education. The emergence of new technology is not necessarily to replace the original technology, but to complement and balance each other. Cloud computing and big data which have the features of centralization, and the blockchains which have the features of decentralization can achieve the balance of yin and Yang, one is in the other, complement each other. In addition, the autonomy of the system is not equal to anarchy or no law, but to make the regulation more open, transparent and pluralistic, to make the law more refined and personalized, and let people constantly explore the way which make the law amalgamate into the code. In addition, we should rationally use blockchain technology in the category of human relations, protect the origin and source of education, establish laws and regulations, protect privacy and intellectual property rights, improve people's comprehensive quality and cultivate professional and technical talents. In a word, we should follow the intrinsic and objective law of the development of science and technology so that science and technology can truly benefit humans. 


\section{References}

[1]. Watters Althea Block chain for Education: An Introduction [OL].

[2]. Coindesk.IBM Reveals Proof of Concept for Block-Chain-Powered Internet of Things [EB/OL]. $\mathrm{http} / /$ www.coindesk.com/ibm-reveals-proof-of-concept-for-block-chain-powered-internet-of-th ings/>2017/1/17.

[3]. Jingdiwangtian,Wu Yawned. Blockchain world [M]. Beijing: CITIC Publishing Group, 2016. $5-214$

[4]. Men Zhiyuan,Lu Xiao, Hu Fan gang. Theoretical Path and Application Thinking of Education Reform Driven by Large Data. Journal of Distance Education [J],2017(3).9-18

[5]. Yu Landing Kenyan (Peking University). Blockchain Technology. China Computer Association Communication [J],2017(5).10-15

[6]. Bai Xiaoying,Xing Chunxiao(Tsinghua University). Application of Blockchain in Digital Society. China Computer Association Communication [J],2017(5).36-43

[7]. Zhou Xuefeng,Zhao Zhao(Beijing University). Analytical Computational law. China Computer Association Communication [J],2017(5).29-35

[8]. Xu Tao(Tongji University). Research on The Development Status and Application Value of Blockchain + Education. Journal of Distance Education [J],2017(3).19-28

[9]. Educause.7 Things You Should Know about Block chain [OL]. https://library.eucause.edu/ /media/files/library/2016/9/eli7136.pdf>

[10]. Hagan Learning is Earning [OL].

http://www.iftf.org/future-now/article-detail/learning-is-earing/>

[11]. The Economist. The Trust Machine: The Technology Behind Bit coin Could Transform How The Economy Works[J]. The Economist,2015,(8):3-9

[12]. Li Liping.Cloud Computing Strategy Analysis in Small and Medium-Sized Enterprises. Proceedings of Science[C]. CeNet2017.524-530 